\title{
PREDICTON OF PERFORMANCE BASED ON DIFFERENT ECONOMIC TRAITS IN DANDARAWI AND GOLDEN MONTAZAH PULLETS.
}

\author{
Ensaf A. El Full and Ali Abd El Azim \\ Department of Poultry Production, Fac. of Agric., Cairo Univ., Fayoum Branch.
}

\begin{abstract}
:
Different prediction equations were established for egg production $\left(\mathrm{EN}_{90}\right)$, egg weight $\left(\mathrm{EW}_{90}\right)$, egg mass $\left(\mathrm{EM}_{90}\right)$ in the first 90 days of production, sexual maturity, specific gravity (SG), shell thickness (ST) and shape index (SI) of egg based on the coefficient values of selected variables under each. Data from Dandarawi (Dand) and Golden Montazah (GM) were used. The R-square values of each equation were calculated. $\mathrm{EN}_{90}$ is a function of either $\mathrm{EW}_{90}$ or $\mathrm{EM}_{90}$ (Dand). $\mathrm{EW}_{90}$ is a function of each of keel length at 8 weeks of age (KL ${ }_{8}$,Dand) and $\mathrm{KL}_{12}$ in GM. SG is a function of ST for both breeds. More $\mathrm{EN}_{90}$ can be predicted through lighter body weight $\left(\mathrm{BW}_{8}\right)$ and longer shank length $\left(\mathrm{SL}_{8}\right)$ at eight weeks of age, however, heavier $\mathrm{BW}_{8}$ and shorter $\mathrm{SL}_{8}$ at eight weeks of age can be used as predictors for higher $\mathrm{EM}_{90}$ for Dand pullets. Heavier body weight at sexual maturity $\left(\mathrm{BW}_{\mathrm{SM}}\right)$ and longer $\mathrm{KL}_{12}$ resulted in retardation of sexual maturation for Dand. The only significant positive factor resulting in $14.5 \%$ of the variability in $\mathrm{EW}_{90}$ was $\mathrm{BW}_{12}$ for $\mathrm{GM}$. Heavier $\mathrm{BW}_{8}$ was associated with decreasing $\mathrm{EN}_{90}$ in Dand. Each of $\mathrm{EN}_{90}, \mathrm{EW}_{90}$ and $\mathrm{BW}_{8}$ were significantly increased $\mathrm{EM}_{90}$ for Dand, whereas $\mathrm{SL}_{8}$ negatively associated with its $\mathrm{EM}_{90}$. For $\mathrm{GM}, \mathrm{EN}_{90}$ and $\mathrm{EW}_{90}$ significantly increased $\mathrm{EM}_{90}$.

In general, equations used for predicting economic traits that had highly significant R-square indicating that variables associated with each $\mathrm{Y}$ trait were actual and prediction for future economic production will be reliable.
\end{abstract}

Key Words: Economic traits, Body weight, Egg production, Egg quality, Dandarawi, Golden Montazah , chickens.

\section{INTRODUCTION}

The economic return of birds depend largely on characters like body weight, age at sexual maturity, egg production, egg weight and other egg quality traits (Singh and Singh, 1999). Rathore et al. (1980) reported that the phenotypic, genetic and environmental correlations between 12 week body weight and egg production were 0.559, 0.152 and 0.053, respectively. Abdel Latif (2001) reported similar findings. According to Singh and Singh (1999), there is a negative correlation between age at sexual maturity and 280day age egg production for White Leghorn. Similarly, genetic correlations are abundant in literature that were calculated between age at sexual maturity and specific gravity of egg, age at sexual maturity and egg mass (Singh and Singh, 1999), egg production and specific gravity (El Full, 1995), egg production and egg weight, egg weight and specific gravity (Abdou $\boldsymbol{e t}$ al., 1993 and Abdel Latif, 2001). However, as shell quality is improved, the breeder must also maintain or improve several other economic traits such as egg production, internal egg quality, fertility, hatchability and viability. Therefore, the choice of shell quality

Fayoum J. Agric. Res. \& Dev., Vol.19, No.1, January, 2005 
measurement should be based, in part, on correlation with these traits (Grunder et al., 1991). Despite of negative correlations between egg number and shell stability, the egg-shell quality could be improved (Pingel et al., 2004). But relationships among these traits with egg production, egg weight, specific gravity of egg, shape index and egg mass are very rare and a better understanding of these relationships is necessary for more effective selection to continue the improvement of the laying hen. Thus present study is an attempt to establish equations for prediction of egg production in the first 90 days of production, egg weight, specific gravity, shape index of egg in Dandarawi and Golden Montazah pullets and to find out the most potent variables associating with each of the above economic traits.

\section{MATERIALS AND METHODS}

The present study was conducted on the Dandarawi (Dand) and Golden Montazah (GM) pullets maintained at El Azab Poultry Research Center, Animal Production Research Institute, Agricultural Research Center, Ministry of Agriculture, Egypt.

A total of 802 female progenies of $\mathrm{G} \mathrm{M}$ and 840 female progenies of Dand breeds were produced using 10 sires per each. Six successive pedigreed hatches, seven days apart were taken. All chicks were wing-banded, immunized for Marek's disease immediately after hatching and brooded in floor brooders. The management practices were kept the same as possible throughout the experimental period. Feed and water were offered $a d$ lib. Feeds formulated and the mineral and vitamins were adequately supplied to cover the requirements of Egyptian chicken breeds according to the Ministerial Decree No. 1498 (1996) .

At 20 weeks of age, the pullets were moved and kept into individual battery cages with standard feeding diets containing $15.5 \% \mathrm{CP}$ and $2700 \mathrm{Kcal}$ $\mathrm{ME} / \mathrm{Kg}$ and management practice.

Individual body weights (BW) at 8, 12 weeks of age and at sexual maturity $\left(\mathrm{BW}_{\mathrm{SM}}\right)$, were recorded to the nearest gram and the lengths of shank and keel (SL and KL) were measured to the nearest $\mathrm{mm}$. Age at sexual maturity $(\mathrm{SM})$ calculated in days per each hen. Egg mass $\left(\mathrm{EM}_{90}\right)$ in grams was obtained by multiplying egg weight $\left(\mathrm{EW}_{90}\right)$ and egg number $\left(\mathrm{EN}_{90}\right)$ laid during the first 90 days of production (up to 265 days of age) for each hen per each breed. The specific gravity (SG) of each egg was estimated using floating technique. The length and breadth of eggs were measured at the first 90 days of egg production at maximum length and width by vernier callipers and expressed as percent ratio of width/length (shape index, SI) of the egg. Shell thickness (ST) was measured on the membraneless shells by using Ames gauge to the nearest $\mu \mathrm{m}$.

Statistical analysis: Data were corrected for hatch effect before conducting the statistical analyses. The following model was used to calculate the descriptive statistical parameters:

where:

$$
Y_{i j k}=\mu+B_{i}+S(B) i j+e_{i j k}
$$

$\mathbf{Y}_{\mathbf{i j k}}$ : expresses the observation of the $\mathbf{i j}^{\text {th }}$ hen within breed and sire, $\boldsymbol{\mu}$ : is the overall mean, $\mathbf{B}_{\mathbf{i}}$ : is the effect of $\mathbf{i}^{\text {th }}$ breed, $\mathbf{S}(\mathbf{B}) \mathbf{i j}$ : is the effect of sire within breed and $\mathbf{e}_{\mathbf{i j k}}$ : is the error term accounted for the $\mathbf{k}^{\mathbf{t h}}$ hen of the $\mathbf{j}^{\mathbf{t h}}$ sire within each $\mathbf{i}^{\text {th }}$ breed .

Fayoum J. Agric. Res. \& Dev., Vol.19, No.1, January, 2005 
The prediction equations based on multiple variables for $\mathrm{EN}_{90}, \mathrm{EW}_{90}$, SG of egg, SI and $\mathrm{EM}_{90}$ were calculated separately through multiple linear and stepwise regression according to SPSS (1998) using the following model: $\hat{\mathbf{Y}}=\mathbf{a}+\mathbf{b}_{1} \mathbf{X}_{1}+\mathbf{b}_{2} \mathbf{X}_{2}+\mathbf{b}_{3} \mathbf{X}_{3}+\ldots . .+b_{k} X_{k}+\varepsilon_{i}$.

where $\hat{\mathbf{Y}}$ : is the expected value or mean of the population of $Y$ 's for a specified set of values of the X's, where: a: represents the Y intercept, b's represents the slopes of $\mathrm{Y}$ on $\mathrm{X}$ that measures the increase or decrease in $\mathrm{Y}$ per unit of $\mathrm{X}$ and $\varepsilon_{i:}$ is a deviation of the observation from the regression line, or a residual. Correlation coefficients among studied traits and variance components were calculated according to SAS (2000).

\section{RESULTS AND DISCUSSION}

Means and their standard errors for BW, SL, KL, egg production related traits and egg quality traits are presented in Table $1 . \mathrm{G} M$ pullets had significantly heavier BW and longer shanks at all studied ages and longer keels at eight weeks of age and at sexual maturity. Lower estimates of BW, SL or KL at 8 and 12 weeks of age for the Dand pullets were reported by Abdel Latif and El Hammady (1992) and Abdel Latif (1999). However, Sabri et al. (1995) reported heavier $\mathrm{BW}_{8}$ and $\mathrm{BW}_{12}$ than the present study for Dand chickens. Dand hens were sexually matured at an earlier age than GM hens by 3.84 days. Earlier age at sexual maturity was reported by Sharaby(1998) and Shebl (1998) for some Egyptian native breeds.

Table 1. Means \pm SE of Dandarawi and Golden Montazah hens for studied traits.

\begin{tabular}{|c|c|c|c|}
\hline Trait & Dandarawi & Golden Montazah & Grand mean \\
\hline $\mathrm{BW}_{8}$ & $466.47 \pm 5.76^{b}$ & $471.46 \pm 1.58^{\mathrm{a}}$ & $468.97 \pm 6.02$ \\
\hline $\mathrm{BW}_{12}$ & $722.59 \pm 10.85^{b}$ & $1039.95 \pm 19.95^{\mathrm{a}}$ & $881.27 \pm 11.35$ \\
\hline $\mathrm{BW}_{\mathrm{SM}}$ & $1256.21 \pm 16.95^{b}$ & $1569.73 \pm 31.16^{\mathrm{a}}$ & $1412.97 \pm 17.74$ \\
\hline $\mathrm{SL}_{8}$ & $75.54 \pm 0.34^{b}$ & $84.94 \pm 0.63^{a}$ & $80.24 \pm 0.36$ \\
\hline $\mathrm{SL}_{12}$ & $89.80 \pm 0.51^{b}$ & $100.61 \pm 0.96^{\mathrm{a}}$ & $95.21 \pm 0.54$ \\
\hline $\mathrm{SL}_{\mathrm{SM}}$ & $92.62 \pm 0.36^{b}$ & $104.78 \pm 0.66^{\mathrm{a}}$ & $98.70 \pm 0.38$ \\
\hline $\mathrm{KL}_{8}$ & $66.08 \pm 1.18^{b}$ & $78.19 \pm 2.02^{\mathrm{a}}$ & $72.14 \pm 1.25$ \\
\hline $\mathrm{KL}_{12}$ & $81.11 \pm 3.98^{\mathrm{a}}$ & $92.31 \pm 7.41^{\mathrm{a}}$ & $86.71 \pm 4.21$ \\
\hline $\mathrm{KL}_{\mathrm{SM}}$ & $96.44 \pm 0.69^{b}$ & $107.19 \pm 1.28^{\mathrm{a}}$ & $101.82 \pm 0.73$ \\
\hline \multicolumn{4}{|c|}{ Egg production-related traits } \\
\hline SM, days & $170.94 \pm 0.46^{b}$ & $174.78 \pm 0.84^{\mathrm{a}}$ & $172.86 \pm 0.48$ \\
\hline $\mathrm{EN}_{90}$ & $64.83 \pm 0.85^{\mathrm{a}}$ & $60.03 \pm 1.55^{b}$ & $62.43 \pm 0.88$ \\
\hline $\mathrm{EW}_{90}, \mathrm{~g}$ & $39.49 \pm 0.27^{b}$ & $48.17 \pm 0.48^{a}$ & $43.84 \pm 0.27$ \\
\hline $\mathrm{EM}_{90}, \mathrm{~g}$ & $2559.41 \pm 37.02^{b}$ & $2888.60 \pm 67.51^{\mathrm{a}}$ & $2724.00 \pm 38.49$ \\
\hline \multicolumn{4}{|l|}{ Egg quality traits } \\
\hline SG & $1.066 \pm 0.001^{\mathrm{a}}$ & $1.062 \pm 0.002^{\mathrm{a}}$ & $1.064 \pm 0.001$ \\
\hline SI, \% & $76.67 \pm 0.26^{\mathrm{a}}$ & $75.82 \pm 0.49^{a}$ & $76.24 \pm 0.28$ \\
\hline $\mathrm{ST}, \mu \mathrm{m}$ & $36.99 \pm 0.28^{\mathrm{a}}$ & $37.28 \pm 0.53^{\mathrm{a}}$ & $37.13 \pm 0.30$ \\
\hline
\end{tabular}

$\mathrm{a}$ and $\mathrm{b}$ : Means with different superscripts in the same row are significantly different $(\mathrm{P} \leq 0.05)$.

Dand hens had significantly $(\mathrm{P} \leq 0.01)$ higher $\mathrm{EN}_{90}$ whereas, GM had highly significant $(\mathrm{P} \leq 0.01)$ heavier $\mathrm{EW}_{90}$ or $\mathrm{EM}_{90}$ as shown in Table 1. Lower egg number during the first 90 days of production was cited by several authors (Sharaby, 1998 and Shebl, 1998). El Hammady et al. (1992) and Ragab (1996) reported heavier egg weight for Dand whereas, lower means of EW was reported by Abdel Galil (1993). Lower egg mass estimates were reported by

Fayoum J. Agric. Res. \& Dev.s Vol.19, No.1, January, 2005 
Sharaby (1998). Eggs produced by Dand pullets had insignificantly higher SG and SI but lower ST than eggs produced by GM pullets.

Variance components as percentage to total variance are presented in Table 2. It can be seen that sire' variance components for Dand BW's at different ages studied were higher than those for GM. Similar trend was obtained for KL's at 8 and 12weeks of age. Higher Dand' variance components due to sires than GM were found for SM, EW 90 , SG, and SI. Whereas, GM had higher sire' variance components than Dand for $\mathrm{SL}_{12}, \mathrm{KL}_{\mathrm{SM}}, \mathrm{EN}_{90}$, and ST. Traits showed highly considerable sire components within breed were affected by breed' genetic makeup than $\mathrm{SL}_{8}$ and SI that affected to a large extent by various environmental factors as shown in Table 2 .

Table 2. Variance components as percent of the total variance for studied traits .

\begin{tabular}{|c|c|c|c|c|}
\hline \multirow{2}{*}{ Item } & \multicolumn{2}{c|}{ Dandarawi } & \multicolumn{2}{c|}{ Golden Montazah } \\
\cline { 2 - 5 } & Sire & Error & Sire & Error \\
\hline $\mathrm{BW}_{8}$ & 25.15 & 74.85 & 22.56 & 77.44 \\
\hline $\mathrm{BW}_{12}$ & 22.28 & 77.72 & 21.80 & 78.20 \\
\hline $\mathrm{BW}_{\mathrm{SM}}$ & 28.38 & 71.62 & 12.47 & 87.53 \\
\hline $\mathrm{SL}_{8}$ & 2.31 & 97.69 & 16.06 & 83.94 \\
\hline $\mathrm{SL}_{12}$ & 7.70 & 92.30 & 8.71 & 91.29 \\
\hline $\mathrm{SL}_{\mathrm{SM}}$ & 14.35 & 85.65 & 12.41 & 87.59 \\
\hline $\mathrm{KL}_{8}$ & 21.34 & 78.66 & 21.29 & 78.71 \\
\hline $\mathrm{KL}_{12}$ & 21.62 & 78.38 & 0.23 & 99.77 \\
\hline $\mathrm{KL}_{\mathrm{SM}}$ & 14.35 & 85.65 & 21.45 & 78.55 \\
\hline $\mathrm{SM}$ & & & & \\
\hline Egg production-related traits & 8.04 & 91.96 & 3.21 & 96.79 \\
\hline $\mathrm{EW}$ & 3.69 & 6.31 & 16.90 & 83.10 \\
\hline $\mathrm{EM}$ & 15.33 & 84.67 & 9.04 & 90.96 \\
\hline Egg quality traits & 6.91 & 93.09 & 5.89 & 94.11 \\
\hline $\mathrm{SG}$ & & & & \\
\hline $\mathrm{SI}$ & 14.78 & 85.22 & 12.31 & 87.6 \\
\hline $\mathrm{ST}$ & 4.25 & 95.75 & 0.62 & 99.38 \\
\hline
\end{tabular}

Phenotypically, SM for Dand positively correlated with $\mathrm{BW}_{\mathrm{SM}}$ and $\mathrm{EW}_{90}\left(0.263, \mathrm{P}<0.01\right.$ and $0.191, \mathrm{P}<0.05$, respectively). $\mathrm{EN}_{90}$ for Dand positively correlated with $\mathrm{EM}_{90}$ however negatively associated with ST (0.894, $\mathrm{P}<0.001$ and $-0.191, \mathrm{P}<0.05$, respectively). $\mathrm{EW}_{90}$ positively correlated with each of $\mathrm{BW}_{8}, \mathrm{BW}_{\mathrm{SM}}, \mathrm{SL}_{\mathrm{SM}}, \mathrm{KL}_{8}, \mathrm{KL}_{\mathrm{SM}}, \mathrm{SM}, \mathrm{EM}_{90}$ and ST whereas negatively correlated with $\mathrm{KL}_{12}$ as shown in Table $3 . \mathrm{EM}_{90}$ significantly correlated with either $\mathrm{EN}_{90}$ or $\mathrm{EW}_{90}$. SG positively correlated with ST $(0.559, \mathrm{P}<0.001)$ however ST was negatively correlated with $\mathrm{EN}_{90}(-0.191, \mathrm{P}<0.05)$. This confirm the views of Singh and Singh (1999).

For GM, negative associations were found between SM and either $\mathrm{BW}_{\mathrm{SM}}$ or $\mathrm{SL}_{\mathrm{SM}}(-0.341$ and $-0.367, \mathrm{P}<0.05)$. $\mathrm{EN}_{90}$ was positively correlated with each of EM and SI $(0.929, \mathrm{P}<0.001$ and $0.448, \mathrm{P}<0.01)$. $\mathrm{GM}^{\prime} \mathrm{EW}_{90}$ was positively correlated with $\mathrm{BW}_{12}, \mathrm{BW}_{\mathrm{SM}}, \mathrm{SL}_{8}, \mathrm{SL}_{\mathrm{SM}}$ and $\mathrm{KL}_{\mathrm{SM}}$ ranging from 0.388 to 0.735 as shown in Table 3. Significant and positive correlation was found between $\mathrm{GM}^{\prime} \mathrm{EM}_{90}$ and either $\mathrm{EN}_{90}$ or SI $(0.929, \mathrm{P}<0.001$ and 0.371, $\mathrm{P}<0.05)$. Similar trend of highly significant positive association was found between SG and ST in GM as in Dand as shown in Table 3.

Fayoum J. Agric. Res. \& Dev., Vol.19, No.1, January, 2005 
Table 3. Phenotypic correlation among various studied traits.

\begin{tabular}{|c|c|c|c|c|c|c|c|}
\hline Item & SM & $\mathbf{E N}_{90}$ & $\mathrm{EW}_{90}$ & $\mathbf{E M}_{90}$ & SG & SI & ST \\
\hline \multicolumn{8}{|c|}{ Dandarawi: } \\
\hline $\mathrm{BW}_{8}$ & 0.116 & -0.132 & $0.325^{* *}$ & 0.044 & $0.180^{*}$ & -0.091 & 0.072 \\
\hline BW $_{12}$ & -0.016 & -0.082 & -0.011 & -0.077 & 0.031 & 0.066 & 0.067 \\
\hline $\mathbf{B W}_{\mathrm{SM}}$ & $0.263 * *$ & -0.164 & $0.430 * *$ & 0.049 & 0.064 & -0.022 & 0.110 \\
\hline $\mathrm{SL}_{8}$ & 0.121 & -0.074 & 0.079 & -0.032 & 0.127 & -0.119 & 0.047 \\
\hline $\mathrm{SL}_{12}$ & 0.125 & -0.106 & -0.172 & -0.177 & 0.025 & 0.020 & 0.096 \\
\hline $\mathbf{S L}_{\mathrm{SM}}$ & 0.069 & -0.071 & $0.314 * * *$ & 0.075 & 0.018 & 0.027 & 0.070 \\
\hline $\mathrm{KL}_{8}$ & -0.029 & -0.057 & $0.203^{*}$ & 0.019 & 0.082 & 0.102 & 0.015 \\
\hline $\mathrm{KL}_{12}$ & 0.096 & 0.013 & $-0.196^{*}$ & -0.092 & -0.091 & -0.071 & -0.003 \\
\hline $\mathbf{K L}_{\mathrm{SM}}$ & 0.108 & -0.063 & $0.232 * *$ & 0.056 & -0.048 & 0.013 & -0.027 \\
\hline SM & & -0.069 & $0.191 *$ & 0.023 & 0.069 & 0.006 & 0.101 \\
\hline $\mathbf{E N}_{90}$ & -0.069 & & -0.036 & $0.894 * * *$ & -0.096 & 0.041 & $-0.191^{*}$ \\
\hline$E_{90}$ & $0.191 *$ & 0.036 & & $0.405 * * *$ & 0.132 & 0.004 & $0.201 *$ \\
\hline $\mathbf{E M}_{90}$ & 0.023 & 0.894 *** & $0.405^{* * *}$ & & -0.025 & 0.040 & -0.079 \\
\hline SG & 0.069 & -0.069 & 0.132 & -0.025 & & 0.061 & 0.559 *** \\
\hline SI & 0.006 & 0.041 & 0.004 & 0.040 & 0.061 & & -0.007 \\
\hline ST & 0.101 & $-0.191^{*}$ & $0.201 *$ & -0.079 & 0.559 *** & -0.007 & \\
\hline \multicolumn{8}{|c|}{ Golden Montazah: } \\
\hline & SM & $\mathbf{E N}_{90}$ & $\mathbf{E W}_{90}$ & $\mathbf{E M}_{90}$ & SG & SI & ST \\
\hline $\mathbf{B W}_{8}$ & -0.026 & -0.075 & 0.309 & 0.045 & 0.128 & -0.270 & 0.187 \\
\hline BW $_{12}$ & -0.314 & -0.127 & $0.388^{*}$ & 0.021 & $0.338^{*}$ & -0.078 & 0.166 \\
\hline $\mathbf{B W}_{\mathrm{SM}}$ & $-0.341 *$ & -0.234 & $0.668 * * *$ & -0.224 & 0.292 & 0.066 & 0.132 \\
\hline $\mathbf{S L}_{8}$ & -0.225 & -0.076 & $0.350 *$ & 0.049 & 0.09 & -0.104 & 0.187 \\
\hline $\mathbf{S L}_{12}$ & -0.159 & -0.058 & 0.296 & 0.078 & 0.254 & -0.162 & 0.143 \\
\hline $\mathbf{S L}_{\mathrm{SM}}$ & $-0.367 *$ & 0.047 & $0.735 * * *$ & 0.138 & 0.095 & -0.016 & 0.103 \\
\hline $\mathrm{KL}_{8}$ & -0.131 & -0.314 & 0.260 & -0.209 & 0.208 & -0.065 & -0.013 \\
\hline $\mathrm{KL}_{12}$ & -0.210 & -0.051 & 0.296 & 0.077 & 0.332 & -0.167 & 0.097 \\
\hline $\mathbf{K L}_{\mathrm{SM}}$ & -0.284 & -0.076 & 0.664 *** & 0.037 & 0.253 & -0.181 & 0.038 \\
\hline SM & & 0.030 & 0.061 & 0.050 & 0.063 & 0.096 & -0.176 \\
\hline $\mathbf{E N}_{90}$ & 0.030 & & -0.082 & $0.929 * * *$ & -0.059 & $0.448^{* * *}$ & 0.054 \\
\hline$E_{90}$ & 0.061 & -0.082 & & 0.287 & 0.151 & -0.148 & 0.080 \\
\hline $\mathbf{E M}_{90}$ & 0.050 & 0.929 *** & 0.287 & & 0.001 & $0.371^{*}$ & 0.080 \\
\hline$\overline{\text { SG }}$ & 0.063 & -0.059 & 0.151 & 0.001 & & 0.077 & 0.467 *** \\
\hline SI & 0.096 & 0.448 ** & -0.148 & $0.371 *$ & 0.077 & & 0.131 \\
\hline ST & -0.176 & 0.054 & 0.080 & 0.080 & $0.467 * *$ & 0.131 & \\
\hline
\end{tabular}

*: Significant at $\mathrm{P} \leq 0.05,{ }^{* *}$ : Significant at $\mathrm{P} \leq 0.01, * * *$ : Significant at $\mathrm{P} \leq 0.001$.

From Table 4, multiple linear regression analysis clearly showed that $\mathrm{BW}_{8}$ for Dand had significant $(\mathrm{P} \leq 0.05)$ negative influence on $\mathrm{EN}_{90}$, perhaps the 8 week age has a significant effect on this economic trait. Whereas, $\mathrm{BW}_{8}$ significantly $(\mathrm{P} \leq 0.05)$ increased $\mathrm{EM}_{90}$ and $\mathrm{SG}$. This confirm the results of Singh and Singh (1999) that egg production was a function of body weight in White Leghorn. 
Table 4. Multiple linear regression for Dandarawi traits.

\begin{tabular}{|l|c|c|c|c|c|c|c|}
\hline \multicolumn{1}{|c|}{ Item } & $\mathbf{S M}$ & $\mathbf{E N}_{\mathbf{9 0}}$ & $\mathbf{E W}_{\mathbf{9 0}}$ & $\mathbf{E M}_{\mathbf{9 0}}$ & $\mathbf{S G}$ & $\mathbf{S I}$ & $\mathbf{S T}$ \\
\hline $\mathbf{a}$ & 115.32 & 54.34 & 35.61 & -2077.50 & 0.95 & 55.85 & -107.60 \\
\hline $\mathbf{B W}_{\mathbf{8}}$ & $-1.53 \mathrm{E}-02$ & $-6.51 \mathrm{E}-03 *$ & $-2.91 \mathrm{E}-03$ & $0.25 *$ & $8.42 \mathrm{E}-05^{*}$ & $-5.71 \mathrm{E}-03$ & $-1.58 \mathrm{E}-02$ \\
\hline $\mathbf{B W}_{\mathbf{1 2}}$ & $8.19 \mathrm{E}-04$ & $-6.82 \mathrm{E}-04$ & $-4.01 \mathrm{E}-04$ & $2.27 \mathrm{E}-02$ & $4.73 \mathrm{E}-07$ & $1.99 \mathrm{E}-03$ & $1.05 \mathrm{E}-03$ \\
\hline $\mathbf{B W}_{\mathbf{S M}}$ & $1.43 \mathrm{E}-02 * *$ & $-8.80 \mathrm{E}-04$ & $-1.37 \mathrm{E}-04$ & $2.86 \mathrm{E}-02$ & $-1.09 \mathrm{E}-05$ & $2.88 \mathrm{E}-05$ & $2.36 \mathrm{E}-03$ \\
\hline $\mathbf{S L}_{\mathbf{8}}$ & 0.19 & $6.29 \mathrm{E}-02$ & $2.16 \mathrm{E}-02$ & -2.37 & $8.38 \mathrm{E}-06$ & -0.19 & $3.51 \mathrm{E}-02$ \\
\hline $\mathbf{S L}_{\mathbf{1 2}}$ & $8.77 \mathrm{E}-02$ & $-8.63 \mathrm{E}-03$ & $-8.54 \mathrm{E}-03$ & 0.27 & $-1.56 \mathrm{E}-05$ & $4.76 \mathrm{E}-02$ & $4.00 \mathrm{E}-02$ \\
\hline $\mathbf{S L}_{\mathbf{S M}}$ & -0.25 & $4.46 \mathrm{E}-02$ & $-2.36 \mathrm{E}-02$ & -1.63 & $-1.23 \mathrm{E}-04$ & $7.35 \mathrm{E}-02$ & $4.83 \mathrm{E}-02$ \\
\hline $\mathbf{K L}_{\mathbf{8}}$ & -0.13 & $2.54 \mathrm{E}-02$ & $2.51 \mathrm{E}-02$ & -1.03 & $3.28 \mathrm{E}-04$ & $8.87 \mathrm{E}-02$ & $-6.56 \mathrm{E}-02$ \\
\hline $\mathbf{K L}_{\mathbf{1 2}}$ & 0.11 & $1.61 \mathrm{E}-02$ & $9.59 \mathrm{E}-03$ & -0.64 & $-1.09 \mathrm{E}-04$ & $-6.75 \mathrm{E}-02$ & $2.58 \mathrm{E}-02$ \\
\hline $\mathbf{K L}_{\mathbf{S M}}$ & $5.72 \mathrm{E}-02$ & $-2.86 \mathrm{E}-02$ & $-2.36 \mathrm{E}-02$ & 1.12 & $-4.09 \mathrm{E}-04$ & $3.81 \mathrm{E}-03$ & $-1.19 \mathrm{E}-02$ \\
\hline $\mathbf{S M}$ & --- & $-1.05 \mathrm{E}-03$ & $4.26 \mathrm{E}-03$ & $3.25 \mathrm{E}-02$ & $1.28 \mathrm{E}-04$ & $2.51 \mathrm{E}-02$ & $-1.05 \mathrm{E}-02$ \\
\hline $\mathbf{E N}_{\mathbf{9 0}}$ & $-2.93 \mathrm{E}-02$ & --- & $-0.62 * * *$ & $38.32 * * *$ & $2.39 \mathrm{E}-04$ & $-6.34 \mathrm{E}-02$ & -0.32 \\
\hline $\mathbf{E W}_{\mathbf{9 0}}$ & 0.29 & $-1.49 * * *$ & --- & $58.02 * * *$ & $2.02 \mathrm{E}-04$ & -0.167 & -0.19 \\
\hline $\mathbf{E M}_{\mathbf{9 0}}$ & $6.10 \mathrm{E}-04$ & $2.58 \mathrm{E}-02 * * *$ & $1.62 \mathrm{E}-02$ & --- & $-5.44 \mathrm{E}-06$ & $2.12 \mathrm{E}-03$ & $6.90 \mathrm{E}-03$ \\
\hline $\mathbf{S G}^{2}$ & 23.11 & 1.54 & 0.54 & -52.21 & --- & 25.91 & $146.72 * * *$ \\
\hline SI & $5.15 \mathrm{E}-02$ & $-4.66 \mathrm{E}-03$ & $-5.09 \mathrm{E}-03$ & 0.23 & $2.94 \mathrm{E}-04$ & --- & $-4.29 \mathrm{E}-02$ \\
\hline ST & $-2.74 \mathrm{E}-02$ & $-2.99 \mathrm{E}-02$ & $-7.16 \mathrm{E}-03$ & 0.96 & $2.12 \mathrm{E}-03 * * *$ & $-5.46 \mathrm{E}-02$ & --- \\
\hline $\mathbf{R}^{\mathbf{2}}$ & 0.172 & 0.991 & 0.960 & 0.992 & 0.375 & 0.071 & 0.388 \\
\hline
\end{tabular}

*: Significant at $\mathrm{P} \leq 0.05$, **: Significant at $\mathrm{P} \leq 0.01, * * *$ : Significant at $\mathrm{P} \leq 0.001$.

A significant effect of $\mathrm{BW}_{\mathrm{SM}}(\mathrm{P} \leq 0.01)$ on $\mathrm{SM}$ was found. A significant $(\mathrm{P} \leq 0.001)$ mutually negative effect persists between $\mathrm{EN}_{90}$ and $\mathrm{EW}_{90}$. However, $\mathrm{EM}_{90}$ significantly $(\mathrm{P} \leq 0.001)$ increased by $\mathrm{EN}_{90}$ and $\mathrm{EW}_{90}$. Singh and Singh (1999) illustrated that the effect of egg weight on egg production is negative indicating that more egg production resulted in loss of weight of egg. ST increased significantly $(\mathrm{P} \leq 0.001)$ with $\mathrm{SG}$.

As shown in Table 5, multiple linear regression analysis indicated that $\mathrm{SL}_{12}$ for GM pullets negatively $(\mathrm{P} \leq 0.01)$ affected either $\mathrm{EN}_{90}$ or $\mathrm{EW}_{90}$ however, significantly $(P \leq 0.01)$ increased $\mathrm{EM}_{90}$. It is clear that $\mathrm{EN}_{90}$ significantly decreased $\mathrm{EW}_{90}$ whereas $\mathrm{EW}_{90}$ significantly $(\mathrm{P} \leq 0.01)$ decreased $\mathrm{EN}_{90}$ but increased $(\mathrm{P} \leq 0.001) \mathrm{EM}_{90}$. Consequently, significant $(\mathrm{P} \leq 0.001)$ positive effects were shown for $\mathrm{EM}_{90}$ on $\mathrm{EN}_{90}$ and $\mathrm{EW}_{90}$. ST of GM' egg increased significantly $(\mathrm{P} \leq 0.01)$ with SG. Similar trends were reported by Petec et al. (2000) for a commercial layer flock and Amardeep et al. (2001) in two White Leghorn strains that shank length significantly affected layer performance and would be a very useful method for estimating pullet quality.

From stepwise regression results presented in Table 6 after excluding insignificant factors, it is clear that $\mathrm{EN}_{90}$ for Dand significantly affected by $\mathrm{EM}_{90,} \mathrm{EW}_{90}, \mathrm{BW}_{8}, \mathrm{SL}_{8}$ with $\mathrm{R}^{2}$ of 0.990 . It can be concluded that more $\mathrm{EN}_{90}$ can be predicted through lighter $\mathrm{BW}_{8}(\mathrm{P} \leq 0.001)$ and longer $\mathrm{SL}_{8}(\mathrm{P} \leq 0.01)$. However, higher $\mathrm{EM}_{90}$ can be expected through higher $\mathrm{EN}_{90}$, heavier $\mathrm{EW}_{90}$ and $\mathrm{BW}_{8}$ associated with shorter $\mathrm{SL}_{8}\left(\mathrm{R}^{2}=0.991\right)$. Heavier $\mathrm{BW}_{8}(\mathrm{P} \leq 0.001)$ and shorter $\mathrm{SL}_{8}(\mathrm{P} \leq 0.01)$ can be used as predictors for higher $\mathrm{EM}_{90}$ for Dand. Similar finding was reported by Petec et al. (2000) that as SL increased EN and EM increased. Using $\mathrm{EM}_{90}, \mathrm{EN}_{90}$ and $\mathrm{KL}_{8}$ as predictors resulted in 0.956 of variations in $\mathrm{EW}_{90}$ for Dand. Longer $\mathrm{KL}_{8}$ can be used as a predictor to heavier

Fayoum J. Agric. Res. \& Dev., Vol.19, No.1, January, 2005 
PREDICTON OF PERFORMANCE BASED ON DIFFERENT.

$\mathrm{EW}_{90}$. ST resulted in $31.1 \%$ of variability in SG, however either EN or SG resulted in $33.5 \%$ of the variability in ST of Dand' eggs. It is clear that heavier $\mathrm{BW}_{\mathrm{SM}}$ and longer $\mathrm{KL}_{12}$ resulted in retardation of sexual maturation for Dand.

Results of stepwise regression for GM showed that $\mathrm{EM}_{90}$ and $\mathrm{EW}_{90}$ as predictors significantly $(\mathrm{P} \leq 0.001)$ affected $\mathrm{EN}_{90}$ with $\mathrm{R}^{2}$ of 0.996 as shown in Table 7. $\mathrm{EN}_{90}$ and $\mathrm{EW}_{90}$ significantly $(\mathrm{P} \leq 0.001)$ increased $\mathrm{EM}_{90}$ with $\mathrm{R}^{2}$ of 0.996 . The only significant positive factor resulting in $14.5 \%$ of the variability in $\mathrm{EW}_{90}$ was $\mathrm{BW}_{12}$ for GM. Both ST and $\mathrm{KL}_{12}$ significantly $(\mathrm{P} \leq 0.05)$ explained $28.3 \%$ of the variation in SG for GM. The only significant positive factor resulting in $17.9 \%$ of the variability in ST was SG for GM. $\mathrm{EN}_{90}$ significantly increased SI $\left(\mathrm{P} \leq 0.01, \mathrm{R}^{2}\right.$ of 0.208$)$. SL $\mathrm{SL}_{\mathrm{SM}}$ negatively affected $\mathrm{SM}$ for $\mathrm{GM}$ $\left(\mathrm{P} \leq 0.05, \mathrm{R}^{2}=0.137\right)$.

In general, equations used for predicting economic traits that had highly significant $\mathrm{R}$-square indicating that variables caused variation under each $\mathrm{Y}$ trait was actual and prediction for future economic production will be reliable.

Table 5. Multiple linear regression for Golden Montazah traits.

\begin{tabular}{|c|c|c|c|c|c|c|c|}
\hline Item & SM & $\mathbf{E N}_{90}$ & $E W_{90}$ & $\mathbf{E M}_{90}$ & SG & SI & ST \\
\hline a & -78.220 & 14.138 & 12.416 & -699.530 & 0.929 & 55.180 & -176.113 \\
\hline BW $_{8}$ & $-2.19 \mathrm{E}-02$ & $-2.19 \mathrm{E}-03$ & $-1.62 \mathrm{E}-03$ & $9.71 \mathrm{E}-02$ & $1.97 \mathrm{E}-05$ & $-2.49 \mathrm{E}-03$ & -5.70 \\
\hline $\mathbf{B W}_{12}$ & 1.69E-03 & $2.76 \mathrm{E}-04$ & $6.04 \mathrm{E}-04$ & $-2.12 \mathrm{E}-02$ & $1.32 \mathrm{E}-05$ & $6.61 \mathrm{E}$ & -1.32 \\
\hline $\mathbf{B W}_{\mathbf{S M}}$ & $-2.08 \mathrm{E}-02$ & $2.35 \mathrm{E}-04$ & $1.31 \mathrm{E}-04$ & $-1.79 \mathrm{E}-02$ & $1.66 \mathrm{E}-06$ & 6.54 & $5.83 \mathrm{E}$ \\
\hline $\mathrm{SL}_{8}$ & $-2.57 \mathrm{E}-02$ & 4.40E-02 & $3.57 \mathrm{E}-02$ & -1.94 & $-3.72 \mathrm{E}-04$ & $1.13 \mathrm{E}-02$ & 0.14 \\
\hline $\mathbf{S L}_{12}$ & 4.24E-02 & $3.75 \mathrm{E}-02$ & $3.23 \mathrm{E}-02$ & -1.73 & $-7.48 \mathrm{E}-05$ & $2.44 \mathrm{E}-02$ & $4.57 \mathrm{E}-02$ \\
\hline $\mathbf{S L}_{\mathbf{S M}}$ & -0.46 & $8.21 \mathrm{E}-02$ & $6.88 \mathrm{E}-02$ & -3.78 & $-6.07 \mathrm{E}-04^{*}$ & 0.1 & $0.25^{*}$ \\
\hline $\mathrm{KL}_{8}$ & 6.79E-02 & $1.63 \mathrm{E}-02$ & $1.99 \mathrm{E}-02$ & -0.87 & $9.77 \mathrm{E}-05$ & $5.85 \mathrm{E}-02$ & $-8.81 \mathrm{E}-02$ \\
\hline $\mathrm{KL}_{12}$ & 0.29 & -0.12 & -0.10 & $5.73 * *$ & $3.25 \mathrm{E}-04$ & -0. & -0.17 \\
\hline $\mathrm{KL}_{\mathrm{SM}}$ & 0.21 & $-1.49 \mathrm{E}-02$ & $-1.67 \mathrm{E}-02$ & 0. & $2.97 \mathrm{E}-04$ & -0 . & -0 . \\
\hline SM & --- & $1.04 \mathrm{E}-02$ & $1.19 \mathrm{E}-02$ & -0. & $8.83 \mathrm{E}$ & $6.24 \mathrm{E}-02$ & $-1.16 \mathrm{E}-02$ \\
\hline $\mathbf{E N}_{90}$ & 0.65 & -- & $-0.81 * * *$ & 45. & $1.09 \mathrm{E}$ & -0. & -0.45 \\
\hline $\mathrm{EW}_{90}$ & 1.11 & $-1.21 * * *$ & -- & $54.83 * * *$ & $1.09 \mathrm{E}-03$ & -0. & -0.52 \\
\hline $\mathbf{E M}_{90}$ & $-1.59 \mathrm{E}-02$ & $2.19 \mathrm{E}-02^{* * * *}$ & $1.78 \mathrm{E}-02 * * * *$ & --- & $-2.39 \mathrm{E}-05$ & $6.99 \mathrm{E}-03$ & $1.03 \mathrm{E}-02$ \\
\hline SG & 196.77 & 38. & 31. & -1751.99 & --- & 24.86 & $225.14 *$ \\
\hline SI & 0.64 & -3.51 & 2 & & 1.13 & --- & -0.13 \\
\hline ST & -( & & -7.7 & 4.69 & $1.40 \mathrm{E}-$ & -0 . & --- \\
\hline $\mathbf{R}^{2}$ & 0.341 & 0.997 & 0.983 & 0.998 & 0.557 & 0.438 & 0.534 \\
\hline
\end{tabular}

*: Significante at $\mathrm{P} \leq 0.05$, **: Significante at $\mathrm{P} \leq 0.01$, ***: Significante at $\mathrm{P} \leq 0.001$. 
Ensaf A. El Full \& A. Abd El Azim

Table 6. Stepwise regression parameters, coefficient of determination $\left(\mathbf{R}^{2}\right)$, standard error of the estimate (SEE) for predicting different economic traits $(\hat{\mathbf{Y}})$ of Dandarawi pullets.

\begin{tabular}{|c|c|c|c|c|}
\hline Model & $\begin{array}{c}\text { Fitted equation } \\
\hat{\mathbf{Y}}=\mathbf{a}+\mathbf{b}_{1} \mathbf{X}_{1}+\mathbf{b}_{2} \mathbf{X}_{2}+\mathbf{b}_{3} X_{3}+. .+b_{k} X_{k}+\varepsilon_{i} \bullet\end{array}$ & $\mathbf{R}^{2}$ & SEE & Sig. \\
\hline \multicolumn{5}{|l|}{ Dependent variable: $\mathbf{E N}_{90}$} \\
\hline $\begin{array}{l}\text { Predictors: } \\
\qquad \mathrm{EM}_{90} \mathrm{EW}_{90} \mathrm{BW}_{8}, \mathrm{SL}_{8}\end{array}$ & $\begin{array}{l}\hat{\mathrm{Y}}=56.46+2.59 \mathrm{EM}_{90}-1.50 \mathrm{EW}_{90}-9.12 \mathrm{E}- \\
03 \mathrm{BW}_{8}+7.54 \mathrm{E}-02 \mathrm{SL}_{8}\end{array}$ & 0.990 & 0.89 & $\begin{array}{l}* * * \\
* * * \\
* *\end{array}$ \\
\hline \multicolumn{5}{|l|}{ Dependent variable:EM ${ }_{90}$} \\
\hline $\begin{array}{l}\text { Predictors: } \\
\qquad \mathrm{EN}_{90}, \mathrm{EW}_{90}, \mathrm{BW}_{8}, \mathrm{SL}_{8}\end{array}$ & $\begin{aligned} \hat{\mathrm{Y}}=-2151.48 & +38.23 \mathrm{EN}_{90}+57.97 \mathrm{EW}_{90} \\
& +0.34 \mathrm{BW}_{8}-2.89 \mathrm{SL}_{8}\end{aligned}$ & 0.991 & 34.38 & $\begin{array}{l}* * * \\
* * * \\
* *\end{array}$ \\
\hline \multicolumn{5}{|l|}{ Dependent variable:EW $\mathrm{EW}_{90}$} \\
\hline $\begin{array}{l}\text { Predictors: } \\
\qquad \mathrm{EM}_{90}, \mathrm{EN}_{90}, \mathrm{KL}_{8}\end{array}$ & $\begin{aligned} \hat{Y}=36.37 & +1.59 \mathrm{E}-02 \mathrm{EM}_{90}-0.61 \mathrm{EN}_{90} \\
& +2.68 \mathrm{E}-02 \mathrm{KL}_{8}\end{aligned}$ & 0.956 & 0.58 & $\begin{array}{l}* * * \\
* * *\end{array}$ \\
\hline \multicolumn{5}{|l|}{ Dependent variable: SG } \\
\hline Predictors : ST & $\hat{\mathrm{Y}}=0.99+2.09 \mathrm{E}-03 \mathrm{ST}$ & 0.311 & $1.11 \mathrm{E}-02$ & $* * *$ \\
\hline Dependent variable: SI & Variables Entered/Removed & & & \\
\hline \multicolumn{5}{|l|}{ Dependent variable: ST } \\
\hline Predictors : SG, $\mathrm{EN}_{90}$ & $\hat{\mathrm{Y}}=-112.58+144.03 \mathrm{SG}-6.19 \mathrm{E}-02 \mathrm{EN}_{90}$ & 0.335 & 2.92 & $\begin{array}{c}* * * \\
*\end{array}$ \\
\hline \multicolumn{5}{|l|}{ Dependent variable: SM } \\
\hline $\begin{array}{l}\text { Predictors: } \\
\mathrm{BW}_{\mathrm{SM}}, \mathrm{KL}_{12} \\
\end{array}$ & $\hat{\mathrm{Y}}=148.05+1.21 \mathrm{E}-02 \mathrm{BW}_{\mathrm{SM}}+0.13 \mathrm{KL}_{12}$ & 0.115 & 4.68 & $\begin{array}{c}* * * \\
* * \\
\end{array}$ \\
\hline
\end{tabular}

Sig.: significance, $* *$ : significantly different at $\mathrm{P} \leq 0.01, * * *$ : significantly different at $\mathrm{P} \leq 0.001$.

Table 7. Stepwise regression parameters, coefficient of determination $\left(\mathbf{R}^{2}\right)$, standard error of the estimate (SEE) for predicting different economic traits $(\hat{Y})$ of Golden Montazah pullets.

\begin{tabular}{|c|c|c|c|c|}
\hline Model & $\begin{array}{c}\text { Fitted equation } \\
\hat{Y}=\mathbf{a}+\mathbf{b}_{1} \mathbf{X}_{1}+\mathbf{b}_{2} \mathbf{X}_{2}+\mathbf{b}_{3} X_{3}+\ldots+b_{k} X_{k}+\varepsilon_{i} \text {. }\end{array}$ & $\mathbf{R}^{2}$ & SEE & Sig. \\
\hline \multicolumn{5}{|l|}{ Dependent variable: $\mathrm{EN}_{90}$} \\
\hline Predictors: $\mathrm{EM}_{90} \mathrm{EW}_{90}$ & $\mathrm{Y}=56.46+2.59 \mathrm{EM}_{90}-1.50 \mathrm{EW}_{90}$ & 0.996 & 0.74 & $\begin{array}{l}* * * \\
* * *\end{array}$ \\
\hline \multicolumn{5}{|l|}{ Dependent variable:EM ${ }_{90}$} \\
\hline Predictors: $\mathrm{EN}_{90} \mathrm{EW}_{90}$ & $\mathrm{Y}=-2453.76+45.59 \mathrm{EN}_{90}+54.09 \mathrm{EW}_{90}$ & 0.996 & 33.85 & $\begin{array}{l}* * * \\
* * *\end{array}$ \\
\hline \multicolumn{5}{|l|}{ Dependent variable:EW ${ }_{90}$} \\
\hline Predictors: $\mathrm{BW}_{12}$ & $\bar{Y}=35.83+1.19 \mathrm{E}-02 \mathrm{BW}_{12}$ & 0.145 & 3.38 & $*$ \\
\hline \multicolumn{5}{|l|}{ Dependent variable: $\mathrm{SG}$} \\
\hline Predictors : ST, $\mathrm{KL}_{12}$ & $\mathrm{Y}=1.01+9.76 \mathrm{E}-04 \mathrm{ST}+2.51 \mathrm{E}-04 \mathrm{KL}_{12}$ & 0.283 & $3.97 \mathrm{E}-03$ & * \\
\hline \multicolumn{5}{|l|}{ Dependent variable: ST } \\
\hline Predictors : SG & $\bar{Y}=-138.43+165.42 \mathrm{SG}$ & 0.179 & 1.64 & $*$ \\
\hline \multicolumn{5}{|l|}{ Dependent variable: SI } \\
\hline Predictor: $\mathrm{EN}_{90}$ & $\mathrm{Y}=71.23+7.67 \mathrm{E}-02 \mathrm{EN}_{90}$ & 0.208 & 1.71 & ** \\
\hline \multicolumn{5}{|l|}{ Dependent variable: SM } \\
\hline Predictor: & $\mathrm{Y}=219.98-0.43 \mathrm{SL}_{\mathrm{SM}}$ & 0.137 & 5.25 & * \\
\hline
\end{tabular}

Sig.: significance, *: Significant at $\mathrm{P} \leq 0.05$, **: Significant at $\mathrm{P} \leq 0.01$, ***: Significant at $\mathrm{P} \leq 0.001$.

Fayoum J. Agric. Res. \& Dev., Vol.19, No.1, January, 2005 
REFERENCES

Abdel Galil, M. A. (1993). Evaluating the performance of some local breeds of chickens under certain planes of nutrition. Ph. D. Thesis, Fac. of Agric., Minia Univ., Egypt.

Abdel Latif, H.A. (2001). Inheritance of certain plasma constituents and their association with some economic traits in Dandarawi and Golden Montazah hens. M. Sc. Thesis Fac. Agric. Cairo Univ., Egypt.

Abdel Latif, M.A. (1999). Selection for body weight at eight weeks of age in Dandarawi chicken. II- Realized heritabilities and correlated response to selection for growth measurements. Egypt. Poult. Sci. 19: 691-707.

Abdel Latif, M.A. and El Hammady, H. (1992). Heritabilities of some egg production and egg quality traits in Dandarawi chickens. Egypt. Poult. Sci. 12: 751-764.

Abdou, G.A., Harms, R.H. and El Husseiny, O. (1993). Various methods of measuring shell quality in relation to percentage of cracked eggs. Poult. Sci., 72: 2038-2043.

Amardeep, S., Chaudhary, M.L., Brah, G.S. and Sandhu, J.S. (2001). Inheritance of external body measurements and their relationships with economic traits in layer chickens. Indian J. Poult. Sci. 36: 153-157.

El Full, E. A. (1995). Different multivariate approaches for egg production in poultry breeding experiments. Ph. D. Thesis, Fac. Agric. Fayoum, Cairo Univ., Egypt.

El Hammady, H.Y., Sarara, H.H. and El Sheikh, T.M. (1992). Effect of feeding regimens and lighting programs on egg production performance of laying hens. Egypt. Poult. Sci. 12: 791-817.

Grunder, A.A., Fairfull, R.W., Hamilton, R.M.G. and Thompson, B.K. (1991). Correlations between measures of egg shell quality of percentage of intact eggs and various economic traits. Poult. Sci., 70: $1855-1860$.

Ministerial decree No. 1498 (1996). Ministerial decree No. 1498 of the year 1996 concerning Foodstuffs industry, Egypt.

Petec, M., Balci, F., Baspinar, H., Organ, M. and Dikmen, S. (2000). The effects of shank length on some production traits of a commercial layer flock. Veteriner-Fakultesti- Dergisi, -Uludag-Universitesi. 19:129-134.

Pingel, H., Kosba, M.A., Yakout, H. M. and Bahei EI Deen (2004). Situation and prognosis of the global and Egyptian poultry production and breeding. Egypt. Poult. Sci. 24 : 561-570.

Ragab, M.S. (1996). Effect of energy protein restriction on the performance of Dandarawi and Sinai layers. M. Sc. Thesis, Fac. Agric., Fayoum, Cairo Univ., Egypt.

Rathore, D.S., Banerjee, A.K. and Chaudhary, R.P. ( 1980). Note on genetic study on growth and production traits in White Leghorn. Indian J. Anim. Sci.7: 589-592.

Sabri, H.M., Ibrahim, K.A. and Khattab, M.S. (1995). Performance of distinct chicken genotypes fed two levels during the growing period. Egypt. Poult. Sci. 15: 421-443. 
Sharaby, E.O.H. (1998). Comparative study of morphology and productivity for Baladi Behairi and Fayoumi strains. M. Sc. Thesis, Fac. of Agric., Cairo Univ., Egypt.

Shebl, M.K. (1998). Estimation of heritability of clutch size using REML and Henderson 3 and its relationship with growth and egg production traits in chicken. Egypt. Poult. Sci. 18 (I): 167-182.

Singh, B. and Singh, H. (1999). Prediction of performance based on different economic traits in White Leghorn. Indian J. Poult. Sci. 34:77-79.

SAS (2000). SAS User's Guide: Statistics Version 8.1 Edition. SAS Institute Inc., Cary, NC., USA.

SPSS (1998). Statistical Package for Social Sciences. Version 8. SPSS Inc.

التنبؤ بالأداء الإنتاجى للصفات الاقتصادية المختلفة فى دجاج الاندراوي والمنتزه الذهبي

$$
\begin{aligned}
& \text { إنصاف أحمد الفل و على عبد العظيم } \\
& \text { قسم انتاج الدو اجن - كلية الزراعة - جامعة القاهرة الفظ فرع الفيوم. }
\end{aligned}
$$

تم حساب معادلات التنبؤ المختلفة لإنتاج البيض EN

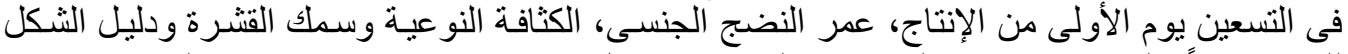

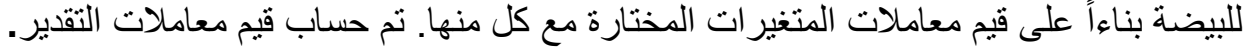
كان

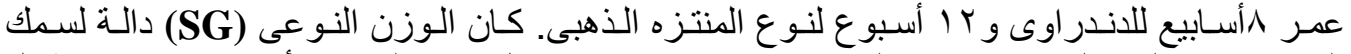
القترة (ST) لكلا النوعين. يمكن التنبؤ بزيـادة

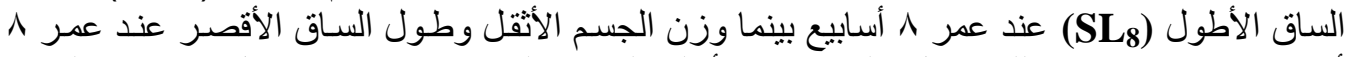

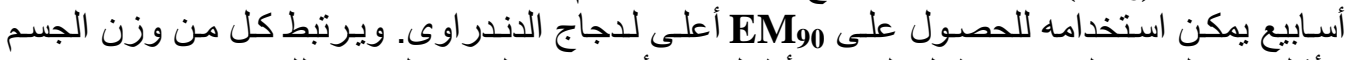

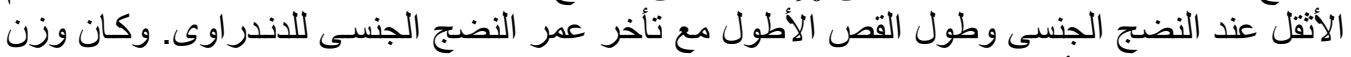

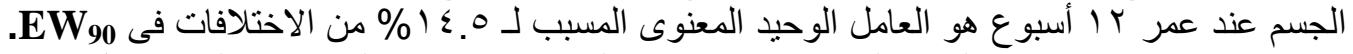

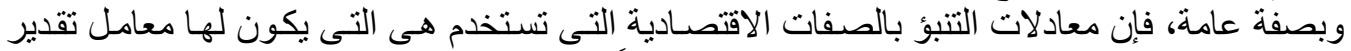

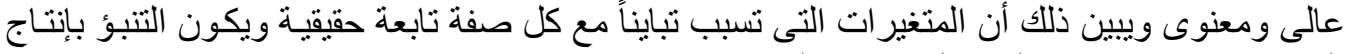
الصفات الاقتصادية فى المستقبل معو لا عليه. 